\title{
ARTIGOS
}

Submetido 02.10.2018. Aprovado 02.08.2019

Avaliado pelo sistema double blind review. Editor Científico: Eduardo Ayrosa

Versão original

DOI: http://dx.doi.org/10.1590/S0034-759020200403

\section{USO DE VIESES COGNITIVOS EM ATIVAÇÕES DE MERCHANDISING E O EFEITO NAS VENDAS NOS SUPERMERCADOS}

\author{
Cognitive biases in merchandising activation and effect on supermarket sales \\ El uso de sesgos cognitivos en activaciones de merchandising y su efecto en \\ las ventas en supermercados
}

\section{RESUMO}

A competição entre marcas disponíveis nos supermercados motiva os esforços das empresas na elaboração e ativação de materiais de comunicação no ponto de venda (MPV). Os MPVs objetivam influenciar o shopper e gerar vendas. A economia comportamental, por sua vez, estuda como um indivíduo toma uma decisão de acordo com as suas próprias preferências e crenças, influenciado pelos vieses cognitivos. 0 objetivo deste trabalho é compreender os efeitos nas vendas a partir do uso dos vieses aversão à perda e contas mentais nas mensagens de materiais de comunicação ativados em supermercados. Para isso, foram realizados dois experimentos de campo aplicados a uma marca de alimento em uma rede de supermercados. Os resultados mostram um efeito positivo e significativo nas vendas apenas quando foi utilizado o viés de aversão à perda na mensagem dos MPVs.

\section{DANTE AVANZI ${ }^{12}$}

dante.avanzi@espm.br

0000-0002-4715-1696

\section{LEANDRO ANGOTTI GUISSONI ${ }^{1}$ \\ leandro.guissoni@fgv.br \\ 0000-0003-1193-9251}

\section{JONNY MATEUS RODRIGUES ${ }^{3}$}

jonnymateus@usp.br

0000-0002-4301-7553

\section{RAFAEL D'ANDREA ${ }^{4}$}

rafael.dandrea@toolboxtm.com.br 0000-0003-3773-4350

${ }^{1}$ Fundação Getulio Vargas, Escola de Administracao de Empresas de Sao Paulo, São Paulo, SP, Brasil

${ }^{2}$ Escola Superior de Propaganda e Marketing, São Paulo, SP, Brasil

3 Universidade de São Paulo, Faculdade de Economia Administração e Contabilidade de Ribeirão Preto, Ribeirão Preto, SP, Brasil

${ }^{4}$ Grupo Toolbox Marketing \& Tech, São Paulo, SP, Brasil
PALAVRAS-CHAVE | Shopper marketing, materiais de comunicação, vieses cognitivos, varejo, economia comportamental.

\section{ABSTRACT}

Competition among different brands in supermarkets motivates consumer goods companies to develop and implement and activate merchandising materials at the point of sales (POS). These merchandising materials attempt to influence shoppers and promote sales. On the other hand, behavioral economics studies the decision-making of individuals based on their preferences and beliefs, influenced by cognitive bias. This study attempts to understand the effect of loss aversion and mental accounting in POS materials on supermarket sales. We conduct two experiments with a consumer brand in a supermarket chain. The results reveal a positive and significant effect on sales only for the cognitive bias of loss aversion, applied to the POS materials.

KEYWORDS / Shopper marketing, merchandising, cognitive bias, retailing, behavioral economics.

\section{RESUMEN}

La competencia entre marcas disponibles en supermercados motiva los esfuerzos de las empresas en el desarrollo y activación de material de comunicación en el punto de venta. Este material de comunicación tiene como meta influenciar shoppers y generar ventas. La economía conductual, a su vez, estudia cómo un individuo toma una decisión de acuerdo con sus propias preferencias y creencias, influenciado por sesgos cognitivos. El objetivo de ese trabajo es comprender los efectos en las ventas a partir del uso de los sesgos cognitivos aversión a la pérdida y contabilidad mental en el material del punto de venta activado en supermercados. Se realizaron dos experimentos con una marca de alimento en una cadena de supermercados. Los resultados revelaron un efecto positivo y significativo en las ventas solo cuando se utilizó el sesgo cognitivo aversión a la pérdida en el material de los puntos de venta.

PALABRAS CLAVE IShopper marketing, material de comunicación, sesgos cognitivos, comercio minorista, economía conductual. 
É muito difícil mudar a forma como o cérebro das pessoas está configurado. Mudar o ambiente e o contexto no qual as pessoas tomam decisões é mais simples. (Gino \& Beshears, 2015, p. 46)

\section{INTRODUÇÃO}

Empresas de bens de consumo investem esforços e recursos para influenciar a decisão do consumidor durante os diversos momentos que formam a jornada de compra de produtos, tendo havido especial atenção para os estímulos de marketing nas lojas físicas (Deloitte, 2009; Shankar, Inman, Mantrala, Kelley, \& Rizley, 2011; Silveira \& Marreiros, 2014). 0 ponto de venda (PDV) tem sido destacado nas pesquisas acadêmicas como importante influenciador na decisão do shopper, principalmente de produtos de consumo não duráveis (Bell, Corsten, \& Knox, 2011; Feijó \& Botelho, 2012; Guissoni, Consoli, \& Rodrigues, 2013; Shankar, 2011; Silveira \& Marreiros, 2014). 0 ambiente de compras nos supermercados influencia o shopper ao ativar o chamado "gatilho de compras" (Löfgren, 2005). Para ativar esse "gatilho de compras" do shopper, os fabricantes de bens de consumo utilizam estímulos de in-store marketing, resultando em ativações de merchandising dentro das lojas para influenciar a tomada de decisão de compra do shopper no PDV (Shankar et al., 2011).

Estudos anteriores mostraram a influência e relevância para as vendas ao investigar diversos estímulos que acontecem no ambiente no PDV. Por exemplo, analisando materiais de comunicação, exposição de produtos, promoções, localização de produtos nas gôndolas e displays (Bell et al., 2011; Chandon, Hutchinson, Bradlow, \& Young, 2009; Court, Elzinga, Mulder, \& Vetvik, 2009; Feijó e Botelho, 2012; Venkatesan, Farris, Guissoni, \& Neves, 2015). Pesquisas sobre o tema também passaram a incorporar elementos da economia comportamental ao utilizar os vieses cognitivos “aversão à perda" (Hardie, Johnson, \& Fader, 1993) e, posteriormente, “contas mentais” (Stilley, Inman, \& Wakefield, 2010a, 2010b). Vieses cognitivos são filtros mentais e emocionais nos quais o indivíduo confia para entender e responder aos eventos externos. Representam, assim, distorções de julgamento que podem afetar a capacidade de um indivíduo de avaliar informações de maneira objetiva e lógica (Tversky \& Kahneman, 1974). Compreendê-los é importante porque os vieses cognitivos influenciam a tomada de decisão de compra e de consumo (Kahneman \& Tversky, 1979; Thaler, 1980).

As pesquisas acadêmicas têm, por sua vez, compreendido a relação dos vieses cognitivos na perspectiva do comportamento do consumidor, e com ênfase dada aos estímulos de promoção de vendas e descontos de preço (Moran \& Montero, 2018; Nunes
\& Park, 2003; Stilley et al., 2010a, 2010b; Thaler, 1985). De maneira geral, tais estudos apresentaram evidências sobre os benefícios do uso desses vieses em estratégias de promoções de vendas e indicaram maiores chances de compras por impulso dos consumidores nos supermercados. Para o viés “aversão à perda", por exemplo, a literatura abordou se shoppers reagiam de maneira diferente se os preços de produtos a eles apresentados estavam posicionados como ganhos ou como perdas (Fabrizi, Lippert, Puppe, \& Rosenkranz, 2016; Kalwani \& Yim, 1992; Kalyanaram \& Winer, 1995). Já para o viés “contas mentais”, estudos acadêmicos trazem os efeitos desse viés quando o shopper aloca diferentes orçamentos mentalmente ao realizar compras de produtos no ambiente de varejo, havendo relação desse viés com as compras por impulso (Reinholtz, Bartels, \& Parker, 2015; Stilley et al., 2010a).

Enquanto os estudos anteriores tradicionalmente investigaram incentivos promocionais aos consumidores utilizando vieses cognitivos específicos (Fabrizi et al., 2016), não há consenso se ativações de merchandising que não ofereçam incentivos de preço poderiam, também, influenciar as vendas de um produto nos supermercados. Stilley et al. (2010b) mencionam que, no campo da economia comportamental aplicada ao shopper marketing, "novas pesquisas poderiam considerar ativações sem promoção de preço” (p. 45). É justamente nessa lacuna que a presente pesquisa pretende contribuir ao investigar se a aplicação de vieses cognitivos em mudanças da mensagem de comunicação de uma marca no PDV (banners e displays), sem um estímulo promocional amplamente presente em estudos anteriores, poderia influenciar as vendas de um produto.

Portanto, a literatura existente sobre o tema não aponta evidências de que os resultados verificados no campo de promoção de preços também seriam aplicados com o uso desses vieses para ativar MPV que não ofereçam estímulos promocionais, importantes nas práticas da indústria e varejo. Na prática empresarial, também tem sido destacada a potencial combinação do tema, isto é, a aplicação de vieses cognitivos a partir de princípios da economia comportamental na comunicação de marketing (Welch, 2010). Assim, a pergunta de pesquisa deste estudo é: Qual o efeito nas vendas a partir da aplicação de premissas dos vieses cognitivos na comunicação e na mensagem de materiais de comunicação em supermercados desenvolvidos para influenciar a decisão de compra do shopper?

Para responder à pergunta de pesquisa, adotou-se uma abordagem de pesquisa quantitativa e experimental, baseada em dois experimentos de campo aplicados a uma marca da categoria de bens de consumo não durável, mais especificamente, de achocolatado em pó, Ovomaltine. 0 experimento foi conduzido em uma rede de supermercados. A partir de entrevistas em 
profundidade com executivos da marca e da revisão de literatura no tema, foram selecionados dois vieses cognitivos, isto é, “aversão à perda" e "contas mentais". Eles são os vieses em que a literatura de varejo tem mostrado interesse por haver implicações já evidenciadas para a prática de shopper marketing no PDV (Stilley et al., 2010a, 2010b). Diferentemente de estudos anteriores que enfatizaram incentivos promocionais, neste estudo, os dois vieses cognitivos foram utilizados na comunicação e na mensagem, por meio de materiais de comunicação, especificamente, em displays especiais e totens de comunicação, sem estímulos promocionais.

\section{FUNDAMENTAÇÃO TEÓRICA E HIPÓTESES}

O tema shopper marketing tem recebido crescente atenção na literatura acadêmica e em publicações de artigos profissionais, especialmente a partir do início de 2005 , com as evidências sobre a importância da decisão do shopper no momento que está no PDV e durante sua jornada de compras (Chandon et al., 2009; Court et al., 2009; Löfgren, 2005; Shankar et al., 2011). 0 presente estudo está relacionado com a ênfase de pesquisa em shopper marketing sobre os fatores e estímulos de marketing que acontecem dentro do ambiente de loja, como ativações de merchandising, que tradicionalmente têm recebido destaque na literatura (Chandon et al., 2009; Shankar et al., 2011; Venkatesan, Farris, Guissoni, \& Neves, 2015) a partir do chamado “primeiro momento da verdade", isto é, o momento que o shopper está em frente às gôndolas e toma uma decisão de compra (Silveira \& Marreiros, 2014; Stilley et al., 2010b).

Neste estudo, foram utilizados vieses cognitivos em materiais de comunicação para analisar a influência das vendas aos consumidores a partir dos fundamentos de shopper marketing. Vieses cognitivos são filtros mentais e emocionais nos quais o indivíduo incorre para entender e responder aos eventos externos, e afetam a tomada de decisão objetiva e lógica (Tversky \& Kahneman, 1974). Quando aplicados nas ativações nos no PDV, os vieses cognitivos podem influenciar o shopper na escolha de marcas adquiridas (Wansink, Kent, \& Roch, 1998). Nesse sentido, os vieses "aversão à perda" e "contas mentais", escolhidos para o estudo, têm sido enfatizados na literatura de varejo (Stilley et al., 2010a, 2010b).

\section{0 viés cognitivo "aversão à perda"}

A teoria da perspectiva surgiu com a proposta de estudar como os indivíduos decidem entre alternativas que ofereçam riscos em relação ao uso do dinheiro (Kahneman \& Tversky, 1979). Um dos seus princípios é representado pelo viés cognitivo "aversão à perda", no qual, quando diretamente comparadas ou ponderadas, o impacto das perdas se mostra maior que os ganhos de mesma magnitude (Kahneman \& Tversky, 1979; Thaler, 1980). Desde então, pesquisadores têm estudado a aversão à perda em múltiplas aplicações e contextos. No varejo, por exemplo, ao comparar o preço de um produto com o seu preço de referência, o consumidor percebe a variação dos preços como "ganhos" ou “perdas”, sendo a sensibilidade à percepção de perda maior do que ao ganho de mesma intensidade (Fabrizi et al., 2016; Hardie et al.,1993; Mayhew \& Winer, 1992; Putler, 1992).

Foi também verificado que o uso de aversão à perda pode despertar sentimentos e emoções (Novemsky \& Kahneman, 2005). Por exemplo, o sentimento de culpa no consumidor pode influenciar a decisão de compra de um produto indulgente ou que ele de fato não precisaria, mas que foi influenciado pela comunicação persuasiva em uma campanha de marketing que enfatizou uma possível perda se ele não comprasse o produto (Burnett \& Lunsford, 1994; Han, Duhachek, \& Agrawal, 2014; Huhmann \& Brotherton, 1997). No entanto, tais estudos abordaram estímulos relacionados ao valor do dinheiro, como preço e promoção. Neste estudo, espera-se que a comunicação apoiada em premissas do viés “aversão à perda" possa resultar em um melhor desempenho de vendas, sem um incentivo promocional, amplamente testado em pesquisas anteriores no tema.

H1: A comunicação elaborada com premissas do viés cognitivo "aversão à perda", aplicada em materiais de merchandising em supermercados, causa uma variação positiva e significante nas vendas.

\section{O viés cognitivo "contas mentais" (mental accounting)}

O viés cognitivo “contas mentais" está também associado à teoria da perspectiva. Thaler (1985) mostrou que os consumidores usam uma forma de orçamento mental, ou seja, alocam dinheiro para contas mentais e tentam resistir a novas compras quando o orçamento previsto acaba. Posteriormente, Stilley et al. (2010a) apresentaram evidências de que consumidores têm tais contas mentais durante suas compras visitando supermercados, porém parte das suas contas mentais não é atribuída a um produto específico antes da ida ao PDV, resultando em compras por impulso nos supermercados e que podem ser encorajadas por meio de estímulos promocionais.

Aplicado a produtos de consumo e varejo, neste estudo, investigamos uma aplicação do viés “contas mentais” em shopper marketing: temptation bundling. Tal viés implica uma combinação 
na qual é apresentada ao shopper um estímulo que ofereça um resultado positivo em termos de potencial satisfação, e um outro, negativo, em uma mesma conta mental (Milkman, Minson, \& Volpp, 2013). O resultado negativo está atrelado a uma potencial satisfação instantânea, de curto prazo, que é sobre o que o shopper deseja, mas pode resultar em sentimento de culpa por talvez não ser a melhor opção em termos de benefício de longo prazo. Por sua vez, o resultado positivo está potencialmente vinculado a algo que o shopper deveria fazer, ou seja, não necessariamente é o que deseja, mas traz benefícios de longo prazo, por exemplo, ter uma alimentação saudável. Por meio do viés "temptation bundling", é possível que o shopper consiga reduzir - ou até mesmo cancelar - o resultado negativo resultante de uma satisfação instantânea, devido ao resultado positivo que é apresentado e remete a um benefício de longo prazo na mesma conta mental. Assim, poderá ocorrer uma redução no sentimento de culpa do consumidor (Duke \& Ami, 2018). No estudo, espera-se causar aumento de vendas sem um incentivo promocional, amplamente testado em pesquisas anteriores no tema, utilizando premissas do viés “contas mentais”.

H2: A comunicação elaborada com premissas do viés cognitivo "contas mentais", aplicada em materiais de merchandising em supermercados, causa uma variação positiva e significante nas vendas.

\section{MÉTODO DE PESQUISA}

Para testar as hipóteses de pesquisa, foram realizados dois estudos experimentais envolvendo um cenário de compra de produto no PDV, por meio de um experimento de campo. O teste de $\mathrm{H}_{1}$ foi conduzido para verificar se o efeito "aversão a perda" pode levar ao aumento de vendas, utilizando premissas desse viés para influenciar a decisão do shopper no PDV por meio de MPV sem incentivos promocionais. $\mathrm{O}$ teste de $\mathrm{H} 2$ buscou verificar tal efeito utilizando premissas do viés "contas mentais". Os experimentos foram aplicados a uma marca da categoria "achocolatado em pó", Ovomaltine. A categoria foi escolhida pelo considerável volume de vendas, em torno de $R$ \$1,9 bilhão em 2015 (Nielsen, 2015), e pela baixa interação que o shopper tem com essa categoria em supermercados (Shopper Marketing Group, 2014), o que motiva a mudança necessária no comportamento do shopper na categoria. Tal mudança pode ter seu "gatilho" com a influência de MPV. De maneira secundária, a marca Ovomaltine foi também escolhida pela baixa participação de mercado que ela tem dentro da categoria (Nielsen, 2015). De acordo com Fazio, Powell e Williams (1989) e Nedungadi (1990), dentro de supermercados, shoppers tendem a ser mais influenciados por estímulos de in-store marketing promovidos por marcas com baixa participação de mercado. Isso ocorre porque se pressupõe que marcas com alta participação de mercado investem mais em propaganda e são mais acessíveis na memória do shopper e, portanto, recebem menor atenção em mensagens específicas promovidas por MPV.

Os experimentos foram realizados em uma rede de varejo independente de supermercados com oito lojas no interior de São Paulo, na região da cidade de Mogi Mirim, e divididos em três estágios. No primeiro estágio, todas as lojas tiveram os produtos sem nenhum tratamento (período pré-experimento), isto é, o achocolatado estava disponível para a compra nas prateleiras sem qualquer comunicação entre os dias 13 de março e 30 de abril de 2016. No segundo estágio, todas as lojas foram submetidas a uma semana de pré-testes para validação dos materiais de comunicação e decisão de sua localização dentro das lojas, entre os dias 14 e 22 de maio do mesmo ano. 0 terceiro estágio foi realizado em duas ondas (período pós-experimento), com duração de sete semanas cada uma (início da semana sempre em um domingo), com um intervalo de duas semanas entre elas. A primeira onda, para testar $\mathrm{H}_{1}$ ("aversão à perda"), ocorreu entre os dias 12 de junho e 30 de julho, enquanto a segunda onda, para testar $\mathrm{H}_{2}$ ("contas mentais"), de 14 de agosto a 1 de outubro. $\mathrm{O}$ período de sete semanas por onda foi necessário para ampliar a base amostral, já que a participação de mercado da marca estudada é baixa (Nielsen, 2015), o que reflete dentro da rede de supermercados, local em que o experimento foi realizado. 0 motivo da escolha de o experimento ser aplicado em uma rede de supermercados é em virtude de a literatura ter apresentado um consenso sobre o fato de que shoppers são mais suscetíveis aos estímulos dos fatores de in-store marketing dentro do ambiente de lojas de autosserviço (Bell et al., 2011; Stilley et al., 2010a).

O experimento de campo permite que o pesquisador observe comportamentos significativos e, geralmente, é mais fácil explicar a sua estrutura e os resultados gerados a um público mais amplo (Samson, 2014). Possibilita, também, o exame de relações de causa e efeito, permitindo às empresas melhor compreensão sobre as relações entre uma mudança de estratégia e as reações comportamentais dos seus clientes (Andersen, Ertac, Gneezy, List, \& Maximiano, 2013; Davenport, 2009). Em artigo recente publicado pelo Journal of Marketing Research (Gneezy, 2017), é chamada a atenção para que sejam utilizados experimentos de campo nas pesquisas em marketing. Ao mesmo tempo, são mostradas as dificuldades para realizar pesquisas de campo, como a necessidade de colaboração e aprovação de empresas que abririam possibilidades de intervenções em suas ações conforme recomendações do pesquisador. Neste estudo, foi 
possível a colaboração com empresas que permitissem um experimento de campo para testar as hipóteses do estudo e possibilitassem o seu desenvolvimento.

Assim, o estudo contou com a colaboração de três empresas: (1) rede de supermercados no interior do Estado de São Paulo, com o papel de permitir a ativação dos materiais nas lojas conforme a orientação e acompanhamento do pesquisador; (2) a gestão da marca Ovomaltine no Brasil, com o papel de validar os materiais de acordo com os guias da marca e permitir a intervenção na comunicação de sua marca nos PDV selecionados para a pesquisa; (3) uma agência de shopper marketing responsável pela criação e produção dos materiais a partir das orientações do pesquisador. As Figuras 1 e 2 apresentam a execução de cada um dos tratamentos das duas hipóteses. Com o objetivo de verificar os resultados encontrados para cada uma das hipóteses, foi feito e analisado um tratamento para cada viés cognitivo estudado nos grupos de lojas experimento. Cada uma delas ilustra a ativação dos materiais de comunicação para o grupo de experimento e para o grupo de controle, bem como a localização de todos os materiais aplicados dentro das lojas. Toda a comunicação e mensagem dos materiais foi aprovada pela marca e pela rede de supermercados.

\section{Figura 1. Aversão à perda}

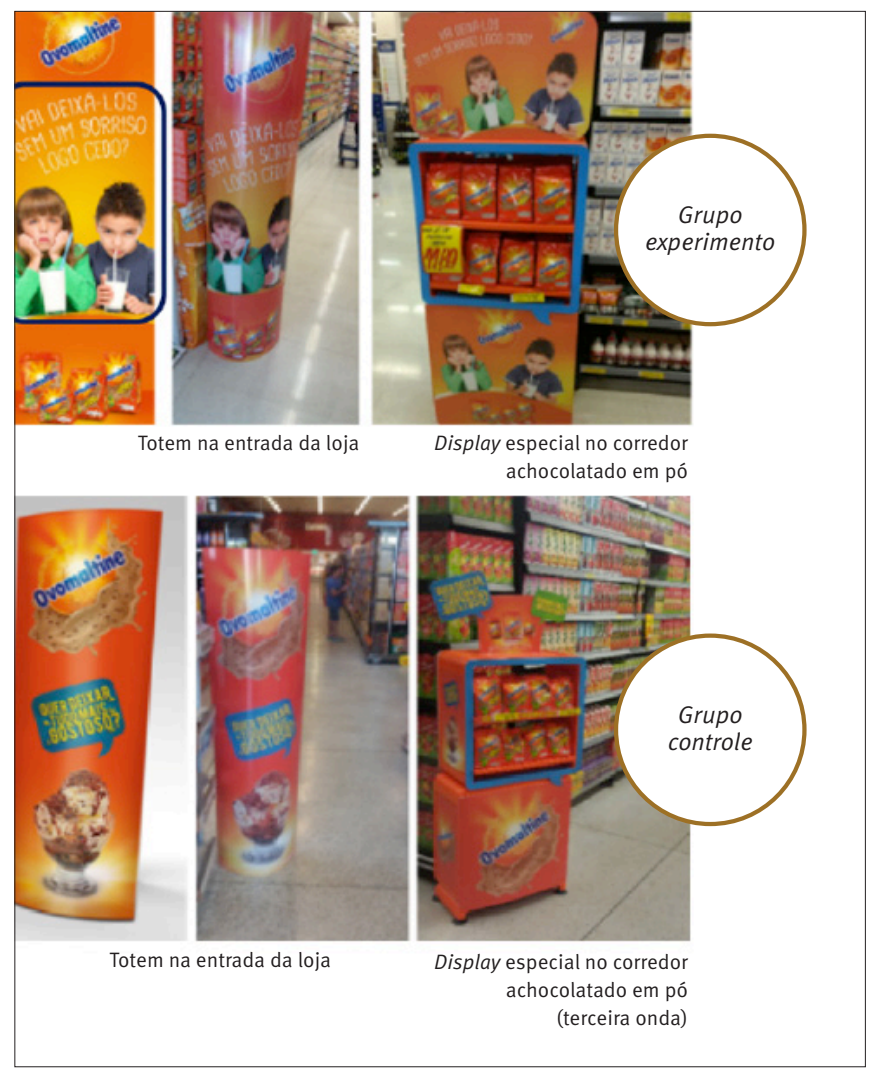

\section{Figura 2. Contas mentais}

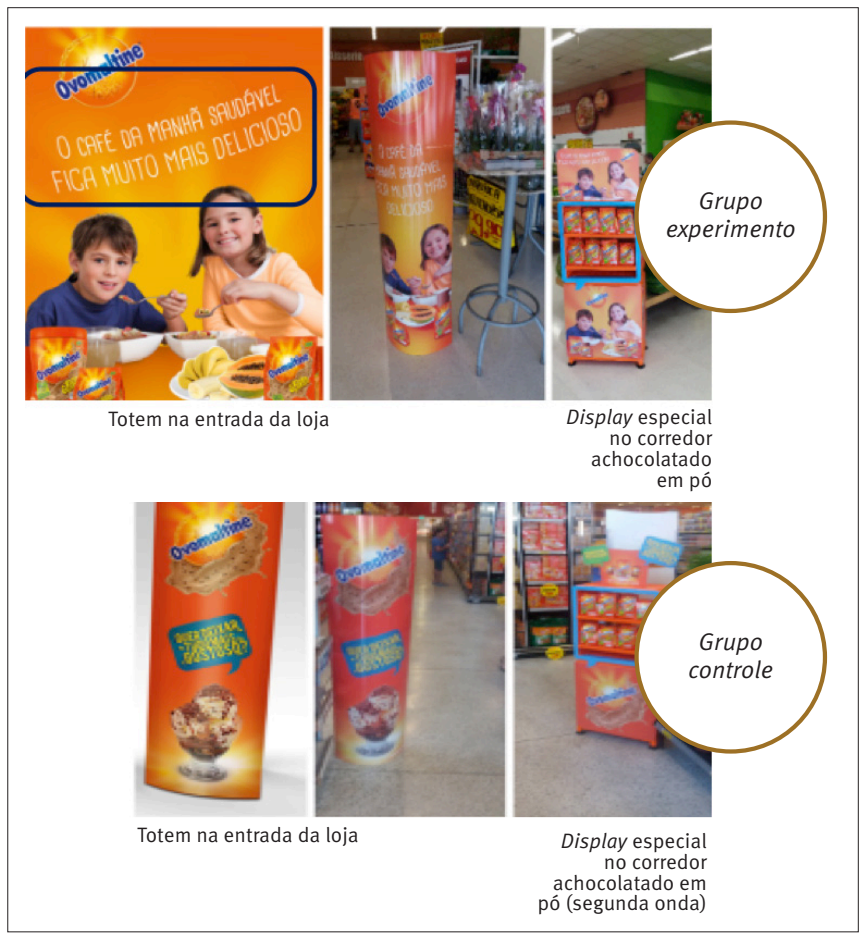

\section{CHECAGEM DE MANIPULAÇÃO DO EXPERIMENTO}

Experimentos de campo são parte da rotina de diversas empresas. Uma vantagem dos experimentos de campo é que os participantes raramente estão cientes de que fazem parte de um experimento, de modo que os dados coletados têm maior probabilidade de representar as realidades predominantes no mercado. Por outro lado, é muito difícil manipular com precisão todos os inputs e ainda controlar variáveis desconhecidas (Venkatesan, Farris, \& Wilcox, 2015, p. 189).

O design do experimento deve garantir a independência das observações e o efeito das variáveis independentes sobre a variável dependente (Price, Jhangiani, Chiang, Leighton, \& Cuttler, 2017), com a condição de isolar a influência de eventos exógenos para estabelecer a relação causal entre as variáveis estudadas. A partir de Venkatesan, Farris e Wilcox (2015), entende-se que uma ação pode ser considerada efetiva quando esta estabelece uma relação causal tal que: o tratamento aumente as vendas do produto; o não uso de material não altere as vendas; o uso do material de comunicação cause efeito nas vendas nos períodos subsequentes; e nenhum outro fator externo contribua para o efeito nas vendas.

Dessa forma, é necessário verificar variáveis que possam ficar confusas ou mesmo estranhas ao experimento. Embora 
fatores externos ao PDV influenciem a tomada de decisão de compra do shopper, como os estímulos de marketing da marca que acontecem fora do ambiente da loja (Bell et al., 2011), eles são isolados no presente trabalho em virtude do foco na comunicação transmitida dentro do ambiente de loja em MPV. Dessa forma, o objetivo do experimento foi observar a variação das vendas da marca levando-se em consideração unicamente o contexto do PDV, por meio da comparação de um grupo de controle com um grupo de experimento, dentro do qual a variável independente, os vieses cognitivos, foi manipulada.

O uso de lojas de controle permite: primeiro, administrar o efeito de crescimento ou queda de vendas do próprio mercado; segundo, verificar se as lojas são semelhantes ou se possuem comportamento distinto de vendas entre elas. 0 uso de vários períodos, assim como um antes e depois, ajuda a satisfazer as condições de causalidade, o aumento no curto prazo e também em períodos subsequentes pode ser verificado. A Tabela 1 apresenta os resultados para as lojas de controle e experimento no Estágio 1, isto é, quando ainda não haviam sofrido o estímulo do tratamento. O procedimento estatístico adotado para a análises das lojas controle e das hipóteses foi embasado na análise de médias de vendas entre os grupos de experimento e de controle. Buscando controlar o efeito que o tamanho das lojas poderia causar nos resultados, as vendas foram analisadas com base na variação semanal em relação à média que a loja realizou.

\section{Tabela 1. Testes comparativos com o grupo de controle} antes do experimento e durante o experimento

\begin{tabular}{c|c|c}
\hline Hipótese & $\begin{array}{c}\text { Antes do experimento, } \\
\text { grupo de experimento } \\
\text { e grupo controle } \\
\text { (Chi-quadrado; } \\
\text { p-valor) }\end{array}$ & $\begin{array}{c}\text { Grupo de controle, } \\
\text { período anterior ao } \\
\text { experimento e durante } \\
\text { experimento }\end{array}$ \\
\hline $\mathrm{H}_{1}$ & 0,$762 ; 0,382$ & 5,$069 ; 0,024^{\star \star}$ \\
\hline $\mathrm{H}_{2}$ & 0,$135 ; 0,713$ & 3,$378 ; 0,066^{\star \star}$ \\
\hline
\end{tabular}

${ }^{(*)}$ Significante para $<<10 \%$; $\left(^{* *}\right)$ Significante para $p<5 \%$; $\left({ }^{* *}\right)$ Significante para $p<1 \%$

Os resultados não apresentam diferenças significativas entre as lojas de controle e as lojas que sofreram os tratamentos. Assim, há evidências de que as lojas não apresentam diferenças significativas em suas vendas. 0 segundo passo é verificar que apenas as lojas do grupo de experimento sofreram aumento significativo de um período para o outro. Os experimentos de campo podem ser facilmente observados pelos concorrentes, e a reação competitiva pode obscurecer os resultados. Mas os experimentos de campo ainda são indicados porque permitem que o profissional de Marketing teste uma campanha com clientes em um ambiente natural (Venkatesan, Farris, \& Wilcox, 2015, p. 189)

Experimentos entre pessoas são frequentemente usados para determinar se um tratamento funciona. Por sua vez, o tratamento é definido como qualquer intervenção destinada a mudar o comportamento da pessoa (Price et al., 2017). No tratamento deste estudo, os fatores de in-store marketing escolhidos para a aplicação dos vieses cognitivos foram materiais de comunicação, especificamente, displays especiais, totens de comunicação e a sua localização dentro da loja, por exercerem forte influência na tomada de decisão do shopper (Inman, Winer, \& Ferraro, 2009; Wilkinson Mason, \& Paksoy, 1982). Para os grupos de experimento, a comunicação e a mensagem, aplicadas nos materiais de comunicação, foram baseadas nas características de cada viés cognitivo abordado nesta pesquisa e conforme hipóteses apresentadas.

As mensagens utilizadas para testar $\mathrm{H}_{1}$ e $\mathrm{H}_{2}$ foram elaboradas pela agência de shopper marketing do grupo Toolbox, tendo o pesquisador acompanhado esse processo. A marca manipulada também disponibilizou pesquisas secundárias da categoria estudada, utilizadas no processo de criação dos materiais de comunicação. As mensagens criadas com premissas dos vieses estudados foram submetidas à avaliação por painel de consumidores na sala de grupo de foco da agência. 0 objetivo foi verificar, de maneira qualitativa, e conforme prática de mercado da empresa que colaborou com o experimento de campo, se as mensagens estavam coerentes com as premissas dos vieses estudados. Em seguida, as mensagens sugeridas pela agência foram submetidas, pelo autor, aos executivos da marca manipulada e aos funcionários do supermercado que realizou o experimento. Com isso, o autor verificou, de maneira qualitativa, apoiado em conversas individuais, se as mensagens estavam coerentes com as ideias desejadas para o teste dos vieses estudados, conforme $\mathrm{H}_{1} \mathrm{e} \mathrm{H} 2$. Por fim, as mensagens finais foram avaliadas pelos autores para garantir a aderência com a literatura relevante do tema, isto é, com as premissas dos vieses. Durante o pré-teste, conforme etapa descrita, o pesquisador também teve conversas individuais com consumidores para poder verificar a aderência dos materiais de comunicação com os vieses estudados, especialmente sobre a percepção de culpa e perda (aversão à perda), e resultados positivos e negativos dentro de uma mesma conta mental (contas mentais). Ademais, os dois materiais, $\mathrm{H}_{1}$ e $\mathrm{H}_{2}$, eram dirigidos aos pais para estimular que comprassem o produto para os filhos, na mesma ocasião de consumo, isto é, café da manhã. Tais decisões seguiram orientações da marca e pesquisas secundárias sobre a categoria, notando que a maioria dos shoppers da categoria "achocolatado 
em pó" compra o produto para o café da manhã dos filhos. As Figuras 1 e 2 mostram as comunicações utilizadas resultantes desse processo. Para o grupo de controle, por outro lado, a comunicação neles aplicada baseou-se na comunicação que já vinha sendo utilizado pela marca estudada e sem premissas ou elementos dos vieses estudados na economia comportamental.

$\mathrm{Na}$ execução do experimento de campo no varejo, entende-se que funcionários de loja mal treinados podem produzir ou interferir, gerando resultados imprecisos. Perturbações são controladas por meio de um plano de mediação específico (Price et al. 2017). Dessa forma, foi realizado treinamento que incluiu o detalhamento técnico do experimento e a conscientização, de todos os gerentes da rede de supermercados, da importância de não interferir diretamente nos resultados dos tratamentos. Para Thomke e Manzi (2014), gerentes e funcionários das lojas podem impactar a homogeneidade dos grupos de experimento, já que, ao estarem cientes dele, podem estar mais ou menos inclinados a melhorar a qualidade do tratamento aplicado. Dessa forma, no presente estudo, a metodologia permitiu compreender a variação nas vendas nos diferentes grupos de lojas (variável dependente) a partir da aplicação dos vieses cognitivos (variável independente), nos materiais de comunicação.

Para buscar a homogeneidade entre os grupos de controle e de experimento, alguns cuidados foram tomados, a saber: (i) as cidades onde estão localizadas as lojas da rede de supermercados são próximas e com um índice IDH-renda muito parecido (Pnud, 2013), o que ajuda a minimizar alguns fatores que pudessem impactar a homogeneidade, como uma alteração climática mais brusca ou um aumento na taxa de desemprego (Moran \& Montero, 2018); (ii) o ticket médio da categoria "achocolatado em pó”, entre todas as oito lojas da rede de supermercado, não apresenta diferença significativa; (iii) com a colaboração da rede varejista durante o experimento de campo, os principais fatores de in-store marketing, no que diz respeito a todas as marcas na categoria "achocolatado em pó", foram controlados, como alterações homogêneas de preços, promoções ou estímulos visuais, garantia de abastecimento dos produtos nas gôndolas em todas as lojas durante todo o período anterior e posterior ao experimento. Os displays especiais e totens de comunicação foram desenvolvidos, exatamente, com as mesmas configurações e dimensões; (iv) o próprio pesquisador acompanhou in loco a evolução dos tratamentos; e (v) realização de treinamentos com todos os gerentes das lojas, antes do início do experimento, e reforços ao longo dele, para garantir a correta execução em todas as lojas. Dessa forma, foram levadas em consideração as seguintes análises de vendas: (1) comparativo da variação da média semanal de vendas, entre os períodos pré e pós-experimento, dentro das lojas do grupo de experimento; (2) comparativo da variação da média semanal de vendas, entre os períodos pré e pós-experimento, entre os grupos de experimento e de controle; e (3) comparativo da variação da média semanal de vendas, entre os períodos pré e pósexperimento, entre a marca estudada e a categoria "achocolatado em pó”, dentro das lojas do grupo de experimento.

\section{ANÁLISE DOS RESULTADOS}

Antes de analisar os comparativos de vendas do procedimento principal e verificar as hipóteses, foi aplicado o teste ShapiroWilk, que é um teste de normalidade. Esse teste é importante para entender se a distribuição dos valores das curvas antes do experimento e das curvas depois do experimento, dos grupos de experimento e de controle, é próxima de uma distribuição normal (Callegari-Jacques, 2003). Para um índice de significância de $10 \%$, todos os testes rejeitam a hipótese nula, o que sugere que não há evidências de que as curvas sigam uma distribuição normal. Portanto, para o procedimento dos testes comparativos de vendas, foi utilizado o teste não paramétrico de análise de médias, Mann-Whitney-Wilcoxon (MWW), que é aplicado para curvas que não tenham distribuição normal.

Outro fator na análise dos dados é que o efeito cumulativo nas vendas pode contaminar os resultados de um experimento. Para evitar esse efeito, deve-se incluir intervalos, sem qualquer intervenção, entre um tratamento e o seguinte (Paksoy, Wilkinson, \& Mason, 1985). Desse modo, para garantir que, no presente estudo, não houvesse efeito cumulativo nas vendas e que os resultados de uma onda não impactassem os resultados de vendas da onda seguinte, elas foram separadas por um intervalo de duas semanas. Esse intervalo é suficiente, já que a frequência média de compra do shopper da categoria "achocolatado em pó" é de uma a duas vezes por mês (Shopper Marketing Group, 2014). Os demais efeitos de covariaveis foram analisados pelo teste de média realizado para a categoria de achocolatados e também entre as lojas de controle pré e pós-experimento; em todos os casos não foram encontradas variações significantes que corroborem a necessidade de correção para sazonalidade e outros efeitos.

\section{TESTE DE H1}

Para a hipótese $\mathrm{H}_{1}$, baseada no viés “aversão à perda”, as perdas assomam como maiores que os ganhos de mesma intensidade (Kahneman \& Tversky, 1979). No tratamento, o objetivo da aplicação dos materiais do grupo de experimento foi explorar na 
comunicação uma situação em que o shopper da categoria achocolatado em pó (neste caso, as mães e pais) perde ao não comprar para os seus filhos o achocolatado em pó da marca estudada. Dessa forma, o material de ativação para testar H1 fortaleceu elementos de perda e culpa, mencionando "vai deixá-los sem um sorriso logo cedo". A Tabela 2 apresenta os resultados das variações de vendas totais, em percentual, encontrados entre o período pré e pós-experimento para o tratamento da hipótese H1 e, também, os $p$-values para os testes de médias não paramétricos.

Tabela 2. Testes comparativos da variação de vendas semanais antes do experimento e depois do experimento para as hipóteses $\mathrm{H}_{1}$ e $\mathrm{H}_{2}$

\begin{tabular}{|c|c|c|c|c|c|c|}
\hline \multirow{3}{*}{$\begin{array}{l}\text { Hipótese e } \\
\text { tratamento }\end{array}$} & \multicolumn{2}{|c|}{ Dentro do grupo de experimento } & \multicolumn{2}{|c|}{$\begin{array}{l}\text { Entre grupo de experimento e } \\
\text { grupo de controle }\end{array}$} & \multicolumn{2}{|c|}{$\begin{array}{l}\text { Grupo do experimento descontando } \\
\text { vendas da categoria, mesmas lojas }\end{array}$} \\
\hline & \multirow{2}{*}{$\begin{array}{c}\text { Variação das } \\
\text { vendas totais (\%) }\end{array}$} & $\begin{array}{l}\text { Teste } \\
\text { MMW }\end{array}$ & \multirow{2}{*}{$\begin{array}{c}\text { Variação das } \\
\text { vendas totais (\%) }\end{array}$} & $\begin{array}{l}\text { Teste } \\
\text { MMW }\end{array}$ & \multirow{2}{*}{$\begin{array}{l}\text { Variação das vendas } \\
\text { totais (\%) }\end{array}$} & Teste MMW \\
\hline & & P-value & & P-value & & P-value \\
\hline $\mathrm{H} 2$ : Contas mentais & $66,2 \%$ & $0,001^{\star \star \star}$ & $22,7 \%$ & 0,312 & $64,8 \%$ & $0,001^{\star \star \star}$ \\
\hline
\end{tabular}

(*) Significante para $p<10 \%$; (**) Significante para $p<5 \%$; $(* *)$ Significante para $p<1 \%$

Para o tratamento da hipótese H1, viés cognitivo “aversão à perda", os resultados dos três testes MMW apresentaram significância, com todos os $p$-values abaixo de $5 \%$. A performance de vendas da marca, dentro do grupo de experimento antes e depois do experimento, foi de $75,4 \%$ (diferenças significantes com $p$-value abaixo de $1 \%)$. A diferença entre as vendas da marca e da categoria foi de $87,7 \%$ ( $p$-value entre o grupo de experimento e o grupo de controle foi de $31,1 \%$ (p-valuero,05).

Dessa forma, com a significância dos resultados dos três testes e a variação positiva das vendas da marca, a hipótese H1 pode ser validada e aceita. Portanto, esse resultado está consistente com a teoria sobre o viés cognitivo "aversão à perda" e suas aplicações ao shopper marketing (Inman et al., 2009; Kahneman, 2011; Mayhew \& Winer, 1992). Mais especificamente, os resultados sugerem, ainda, como o viés “aversão à perda” pode ser efetivo para ativar o "gatilho de compras" do shopper no PDV, ao ser aplicado especificamente em MPVs em supermercados, no “momento da verdade". A aplicação desse viés também foi positiva em uma comunicação que teve como foco uma relação emocional e afetiva, como é a relação de pais com os seus filhos.

\section{TESTE DE H2}

Para a hipótese $\mathrm{H}_{2}$, a premissa do tratamento a partir de premissas do viés cognitivo temptation bundling é apresentar resultados positivos e negativos dentro de uma mesma conta mental (Milkman et al., 2013). A aplicação dos materiais para o grupo de experimento teve como objetivo apresentar a percepção de marca indulgente que a marca estudada tem (Shopper Marketing Group, 2014) como resultado negativo, atrelado ao consumo de frutas saudáveis e nutritivas, apresentado como resultado positivo. A aplicação desse viés cognitivo foi tanto na comunicação dos materiais quanto na localização do display especial, disposto na seção hortifrúti das lojas. Dessa forma, o material de ativação para testar $\mathrm{H}_{2}$ fortaleceu elementos positivos e negativos, mencionando "o café da manhã saudável fica mais delicioso", com a imagem de café da manhã com frutas e, ao mesmo tempo, o achocolatado Ovomaltine (Figura 2). A Tabela 2 apresenta os resultados das variações de vendas encontrados entre o período pré e pós-experimento para o tratamento da hipótese $\mathrm{H} 2$.

Para a hipótese $\mathrm{H}_{2}$, com premissas do viés temptation bundling, as vendas dentro do grupo de experimento cresceram 66,2\% (p-value<o,01) pós-experimento e tiveram um desempenho $22,7 \%$ superior ao grupo de controle, porém não houve significância ( $p$-value de 0,312$)$. Na comparação com as vendas da categoria "achocolatado em pó", dentro do grupo de experimento, a performance de vendas da marca foi $64,8 \%$ $(p<0,01)$ maior do que as vendas da categoria. Dessa forma, a comunicação e a alocação do display especial, na sessão de hortifrúti do supermercado, sugere que o shopper não aloca, na mesma conta mental, a percepção de indulgência, da marca estudada, como resultado negativo (satisfação de curto prazo) e as frutas nutritivas como resultado positivo (benefícios de 
longo prazo). Portanto, não foi possível suportar a H2 e validar a influência nas vendas do uso do viés "contas mentais” nos materiais de comunicação. Uma possível explicação pode estar relacionada com a não utilização de um elemento promocional e evidências do efeito positivo de ativações do tipo cross merchandising que trabalham produtos com propostas de valor parecidas em uma ocasião de consumo (Bell et al., 2011). Por exemplo, descontos e ativações conjuntas oferecidas para o amendoim com a cerveja, em vez de ativações com produtos com benefícios diferentes para balancear as contas mentais do consumidor, por exemplo, um achocolatado em pó e frutas, conforme investigado neste estudo, a partir das premissas do viés contas mentais.

Assim, não foi possível suportar a $\mathrm{H}_{2}$ e validar a influência nas vendas do uso do viés "contas mentais" nos materiais de comunicação, enquanto $\mathrm{H}_{1}$ foi validada. De fato, a literatura validou o efeito de contas mentais no ambiente de supermercados utilizando mais estímulos ligados a um benefício de preço e promoção, enquanto ainda não havia consenso sobre o uso desse viés para trabalhar estímulos sem ativações de preços (Stilley et al., 2010a). Tal literatura sugere que o viés "contas mentais" está associado a uma previsão de orçamento do consumidor, por exemplo, para utilizar em supermercados.

Dessa forma, enfatizar apenas a mensagem na comunicação no PDV, sem um estímulo de preço, parece não ter sido suficiente para observar um efeito positivo e significativo nas vendas. Enquanto isso, a literatura sobre "aversão à perda" aponta evidências de que o viés poderia apresentar um resultado positivo mesmo sem o estímulo do preço (Thaler, 1980), e este estudo corrobora a literatura anterior.

\section{CONCLUSÕES, LIMITAÇÕES E IMPLICAÇÕES GERENCIAIS}

O estudo contribui para mostrar potenciais combinações de shopper marketing e o uso dos vieses cognitivos, base da economia comportamental, na busca de evidências sobre como melhor elaborar MPV para influenciar o shopper. Em complemento aos estudos anteriores que enfatizaram a relação entre os vieses "aversão à perda" e "contas mentais" com incentivos promocionais, o presente estudo investigou a adequação de mensagens veiculadas em MPV sem tais incentivos. Os dois tratamentos do experimento de campo conduzido permitiram observar variações de vendas, complementando estudos anteriores com foco em comportamento do consumidor e promoção de preços a partir de experimentos de laboratório.
Os resultados indicam que o uso do viés cognitivo "aversão à perda" em MPVs apresentou resultado significante e positivo nas vendas, enquanto para o viés “contas mentais" não foi observada tal influência. De fato, como o viés "contas mentais" enfatiza a ideia de orçamento para o consumidor (Thaler, 1985), trabalhar com gatilhos promocionais conforme abordado em estudos anteriores (Stilley et al., 2010a) pode ser mais importante do que haver apenas a mudança de mensagem na comunicação dos MPVs. Ou seja, no uso de premissas do viés "contas mentais", estímulos de preço e promoção não devem ser negligenciados. Por outro lado, o uso de premissas do viés “aversão à perda" apresentou, neste estudo, chances de sucesso quando aplicado com materiais de comunicação sem estímulos de preços. A partir desses resultados, há oportunidades para estudos futuros testarem a relação entre as mensagens nos MPV e preços para vieses cognitivos de interesse.

No tratamento da $\mathrm{H}_{1}$, sobre o viés “aversão à perda”, foram consideradas as relações entre premissas para enfatizar, na comunicação, o sentimento de culpa se o consumidor não comprasse o produto (Novemsky \& Kahneman, 2005), refletido na elaboração dos MPVs. Enquanto isto, no tratamento feito para testar $\mathrm{H} 2$ em relação ao viés contas mentais, foi utilizada a premissa de uma ativação que poderia reduzir o sentimento de culpa do consumidor (Duke \& Ami, 2018). Portanto, baseado nos resultados observados suportando apenas $\mathrm{H}_{1}$, os materiais de comunicação que trabalham enfatizando elementos quanto ao sentimento de culpa podem ter maiores chances de influenciar as vendas sem estímulos de preço do que buscar utilizar elementos que possam minimizar o sentimento de culpa, como foi a premissa para testar a H2. Uma vez que o foco deste estudo foi observar o efeito nas vendas a partir de dois experimentos de campo sobre esses vieses, estudos futuros poderiam investigar tais aspectos de shopper marketing, economia comportamental, risco e culpa com enfoque nos MPVs na perspectiva do comportamento do consumidor. Por exemplo, trabalhando com enfoque na literatura sobre culpa para avaliar as associações dos consumidores com diferentes tipos de comunicação. Futuros trabalhos poderiam, também, considerar outros formatos varejistas com diferentes propostas de valor para o consumidor, como o pequeno varejo, hipermercados e farmácias, ou ainda integrando a comunicação na loja e fora da loja, por exemplo, propaganda em mídias fora do ambiente de loja.

0 estudo apresenta algumas limitações. 0 experimento de campo, pelo acesso do pesquisador, foi realizado com produtos de uma marca com características específicas, conforme já descrito. Desse modo, possíveis pesquisas futuras poderiam ser conduzidas com produtos de outras marcas, de diferentes 
categoriais, e com distintos níveis de participação de mercado. Além disso, o experimento foi realizado em um ambiente de PDV, os supermercados, o que limita a generalização dos resultados e conclusões sobre outros tipos de varejistas. Desenvolver um experimento de campo, como o que foi proposto neste estudo, apresenta vantagens e limitações. 0 pesquisador precisa articular múltiplos interesses de empresas envolvidas, recursos limitados, tempo de executivos e diretrizes de marcas manipuladas, enquanto busca contribuição teórica, rigor metodológico e validação do experimento. Porém, o experimento de campo pode oferecer contribuições importantes ao compreender os efeitos de variáveis de interesse com o que de fato acontece em uma situação real, ou seja, o comportamento em vez de percepções, intenções e atitudes como enfatizado em experimentos de laboratório (Gneezy, 2017).

Como implicações gerenciais, este estudo endereçou oportunidades de pesquisa a partir de uma perspectiva empresarial, por exemplo, identificada pela McKinsey (Welch, 2010), sobre a importância de aprofundar a compreensão do uso dos princípios da economia comportamental em Marketing. Nesse sentido, esta pesquisa pode contribuir com profissionais de bens de consumo e varejistas que buscam novas possibilidades para melhorar os resultados de vendas a partir da ativação de MPV baseado na aplicação de vieses cognitivos em sua comunicação e mensagem. A implicação principal gerencial decorre do fato de que a prática tradicional de comunicação das marcas de consumo não necessariamente é a melhor alternativa. Tradicionalmente, as marcas têm utilizado mensagens genéricas no PDV ou utilizam muitos apelos promocionais. A pesquisa revelou que o uso do viés "aversão à perda" pode influenciar as vendas sem a necessidade de um incentivo promocional que prejudique a margem do varejista e o retorno da indústria de consumo sobre os seus gastos em ativações de merchandising nos supermercados.

\section{REFERÊNCIAS}

Andersen, S., Ertac, S., Gneezy, U., List, J. A., \& Maximiano, S. (2013). Gender, competitiveness, and socialization at a young age: Evidence from a matrilineal and a patriarchal society. Review of Economics and Statistics, 95(4), 1438-1443. doi: 10.1162/REST_a_00312

Bell, D. R., Corsten, D., \& Knox, G. (2011). From point of purchase to path to purchase: How pre shopping factors drive unplanned buying. Journal of Marketing, 75(1), 31-45. doi: 10.1509/jm.75.1.31

Burnett, M. S., \& Lunsford, D. (1994). Conceptualizing guilt in the consumer decision-making process. Journal of Consumer Marketing, 11(3), 33-43. doi: 10.1108/07363769410065454

Callegari-Jacques, S. M. (2003). Bioestatística: Princípios e aplicações. Porto Alegre, RS: Artmed.
Chandon, P., Hutchinson, J. W., Bradlow, E. T., \& Young, S. H. (2009). Does in-store marketing work? Effects of the number and position of shelf facings on brand attention and evaluation at the point of purchase. Journal of Marketing, 73(6), 1-17. doi: 10.1509/jmkg.73.6.1

Court, D., Elzinga, D., Mulder, S., \& Vetvik, O. J. (2009). The Consumer Decision Journey. McKinsey Quarterly. Recuperado de https:// www.mckinsey.com/business-functions/marketing-and-sales/ourinsights/the-consumer-decision-journey

Davenport, T. H. (2009). How to design smart business experiments. Harvard Business Review. Recuperado de https://hbr. org/2009/02/how-to-design-smart-business-experiments

Deloitte. (2009). Shopper marketing: Providing a $360^{\circ}$ view of your shoppers and consumers. London, UK: Deloitte LLP.

Duke, K. E., \& Amir, O. N. (2018). Guilt dynamics: Consequences of temporally separating decisions and actions. Journal of Consumer Research, 45(6), 1254-1273. doi: 10.1093/jcr/ucy049

Fabrizi, S., Lippert, S., Puppe, C., \& Rosenkranz, S. (2016). The manufacturer's suggested retail price. Journal of Economic Psychology, 53, 141-153. doi: 10.1016/j.joep.2016.02.001

Fazio, R. H., Powell, M. C., \& Williams, C. J. (1989). The role of attitude accessibility in the attitude-to-behavior process. Journal of Consumer Research, 16(3), 280-288. doi: 10.1086/209214

Feijó, F. R., \& Botelho, D. (2012). Efeito dos fatores de merchandising nas vendas do varejo. RAE-Revista de Administração de Empresas, 52(6), 628-642. doi: 10.1590/S0034-75902012000600005

Gino, F., \& Beshears, J. (2015). Os líderes como arquitetos de decisão. Harvard Business Review. Recuperado de http://hbrbr.uol.com.br/ os-lideres-como-arquitetos-de-decisao

Gneezy, A. (2017). Field experimentation in marketing research. Journal of Marketing Research, 54(1), 140-143. doi: 10.1509/jmr.16.0225

Guissoni, L. A., Consoli, M. A., \& Rodrigues, J. M. (2013). Is category management in small supermarkets worth the effort?. RAE-Revista de Administração de Empresas, 53(6), 592-603. doi: 10.1590/So03475902013005000005

Han, D., Duhacheck, A., \& Agrawal, N. (2014). Emotions shape decisions through construal level: The case of guilt and shame. Journal of Consumer Research, 41(4), 1047-1064. doi: 10.1086/678300

Hardie, B. G. S., Johnson, E. J., \& Fader, P. S. (1993). Modeling loss aversion and reference dependence effects on brand choice. Marketing Science, 12(4), 378-394. doi: 10.1287/mksc.12.4.378

Huhmann, B. A., \& Brotherton, T. P. (1997). A content analysis of guilt appeals in popular magazine advertisements. Journal of Advertising, 26(2), 33-45. doi: 10.1080/00913367.1997.10673521

Inman, J. J., Winer, R. S., \& Ferraro, R. (2009). The interplay among characteristics, customer characteristics and customer activities on in-store decision making. Journal of Marketing, 73(5), 19-29. doi: 10.1509/jmkg.73.5.19

Kahneman, D. (2011). Thinking, fast and slow. New York, USA: Macmillan.

Kahneman, D., \& Tversky, A. (1979). Prospect theory: An analysis of decision under risk. Econometrica, 47(2), 263-292. doi : $10.2307 / 1914185$

Kalwani, M., \& Yim, C. (1992). Consumer price and promotion expectations: An experimental study. Journal of Marketing Research, 29(1), 90-100. doi: 10.1177/002224379202900108 
Kalyanaram, G., \& Winer, R. S. (1995). Empirical generalizations from reference price research. Marketing Science, 14(3), G1-G236. doi: $10.1287 / \mathrm{mksc} .14 \cdot 3 \cdot \mathrm{G}_{161}$

Löfgren, M. (2005). Winning at the first and second moments of truth: An exploratory study. Managing Service Quality, 15(1), 102-115. doi: $10.1108 / 09604520510575290$

Mayhew, G. E., \& Winer, R. S. (1992). An empirical analysis of internal and external reference prices using scanner data. Journal of Consumer Research, 19(1), 62-71. doi: 10.1086/209286

Milkman, K. L., Minson, J. A., \& Volpp, K. G. M. (2013). Holding the hunger games hostage at the gym: An evaluation of temptation bundling. Management Science, 6o(2), 283-299. doi: 10.1287/mnsc.2013.1784

Moran, D., \& Montero, M. (2018) Automated behavioral economics patterns in promotion testing and methods therefor. U.S. Patent Application No. 10,140,629. Washington, DC: U.S. Patent and Trademark Office.

Nedungadi, P. (1990). Recall and consumer consideration sets: influencing choice without altering brand evolutions. Journal of Consumer Research, 17(3), 263-276. doi: 10.1086/208556

Nielsen. (2015). Nielsen Retail Index. Seminário Nielsen Tendências, São Paulo, SP.

Novemsky, N., \& Kahneman, D. (2005). The boundaries of loss aversion. Journal of Marketing Research, 42, 119-128. doi: 10.1509/ jmkr.42.2.119.62292

Nunes, J. C., \& Park, C. W. (2003). Incommensurate resources: Not just more of the same. Journal of Marketing Research, 40, 26-38. doi: 10.1509/jmkr.40.1.26.19131

Paksoy, C. H., Wilkinson, J. B., \& Mason, J. B. (1985). Learning and carryover effects in retail experimentation. Journal of the Market Research Society, 27(2), 109-129.

PNUD. (2013). Ranking IDH entre os municípios. Recuperado de http:// www.pnud.org.br/atlas/ranking/Ranking-IDHM-Municipios-2010. aspx

Price, P. C., Jhangiani, R. S., Chiang, I. A., Leighton, D. C., \& Cuttler, C. (2017). Research methods in psychology ( $3^{\text {rd }}$ ed.). Pullman, USA: Open Text WSU.

Putler, D. S. (1992). Incorporating reference price effects into theory of consumer choice. Marketing Science, 11(3), 287-309. doi: 10.1287/ mksc.11.3.287

Reinholtz, N., Bartels, D. M., \& Parker, J. R. (2015). On the mental accounting of restricted-use funds: How gift cards change what people purchase. Journal of Consumer Research, 42, 596-614. doi: $10.1093 / \mathrm{jcr} / \mathrm{ucv0} 45$

Samson, A. (2014). The Behavioral Economics Guide 2014. London, UK: Behavioral Economics Group.
Shankar, V. (2011). Shopper marketing. Massachussets, USA: Marketing Science Institute.

Shankar, V., Inman, J. J., Mantrala, M., Kelley, E., \& Rizley, R. (2011). Innovations in shopper marketing: Current insights and future research issues. Journal of Retailing, 87(Supplement 1), S29-S42. doi: 10.1016/j.jretai.2011.04.007

Shopper Marketing Group. (2014). Understanding the chocolate powder shopper. São Paulo: ShopperMkt.

Silveira, P.D., \& Marreiros, C. G. (2014). Exploring shopper marketing approach implications on brand communication at the point-ofpurchase: An expert's opinion qualitative study. The Journal of Applied Business Research, 30(5), 1329-1338.

Stilley, K. M., Inman, J.J., \& Wakefield, K. L. (2010a). Planning to make unplanned purchases? The role of in store slack in budget deviation. Journal of Consumer Research, 37(2), 264-278. doi: 10.1086/651567

Stilley, K. M., Inman, J. J., \& Wakefield, K. L. (2010b, May). Spending on the fly: Mental budgets, promotions, and spending behavior. Journal of Marketing, 74 , 34-47. doi: 10.1509/jmkg.74.3.034

Thaler, R. H. (1980). Toward a positive theory of consumer choice. Journal of Economic Behavior \& Organization, 1(1), 39-60. doi: 10.1016/01672681(80)90051-7

Thaler, R. H. (1985). Mental accounting and consumer choice. Marketing Science, 27(1), 15-25. doi: 10.1287/mksc.1070.0330Thomke, S., \& Manzi, J. (2014). The discipline of business experimentation. Harvard Business Review. Recuperado de https://hbr.org/2014/12/thediscipline-of-business-experimentation

Tversky, A., \& Kahneman, D. (1974). Judgment under uncertainty: Heuristics and biases. Science, 185(4157), 1124-1131. doi: 10.1126/ science.185.4157.1124

Venkatesan, R., Farris, P., Guissoni, L. A., \& Neves, M. F. (2015). Consumer brand marketing through full-and self-service channels in an emerging economy. Journal of Retailing, 91(4), 644-659. doi: 10.1016/j.jretai.2015.04.003

Venkatesan, R., Farris, P., \& Wilcox, R. T. (2015). Cutting-edge marketing analytics: Real world cases and data sets for hands on learning. Upper Saddle River, New Jersey: Pearson Education.

Wansink, B., Kent, R. J., \& Hoch, S. J. (1998). An anchoring and adjustment model of purchase quantity decisions. Journal of Marketing Research, 35(1), 71-81. doi: 10.1177/002224379803500108

Welch, N. (2010). A marketer's guide to behavioral economics. McKinsey Quarterly, 47(1), 1-4.

Wilkinson, J., Mason, J., \& Paksoy, C. (1982). Assessing the impact of short term supermarket strategy variables. Journal of Marketing Research, 19(1), 72-86. doi: 10.1177/002224378201900107

\section{CONTRIBUIÇÃO DOS AUTORES}

Os autores declaram que participaram de forma conjunta em todas as etapas de desenvolvimento do texto. Desde a conceitualização e abordagem teórica-metodológica, coleta de dados, bem como a revisão teórica (levantamento de literatura) e por fim, redação e revisão final do artigo. 